\title{
BMJ Open Cluster randomised controlled trial of a consumer behaviour intervention to improve healthy food purchases from online canteens: study protocol
}

Tessa Delaney, ${ }^{1,2,3,4}$ Rebecca Wyse, ${ }^{1,2,3,4}$ Sze Lin Yoong, ${ }^{1,2,3,4}$ Rachel Sutherland, ${ }^{1,2,3,4}$ John Wiggers, ${ }^{1,2,3,4}$ Kylie Ball, ${ }^{5}$ Karen Campbell, ${ }^{5}$ Chris Rissel, ${ }^{6}$ Luke Wolfenden ${ }^{1,2,3,4}$

To cite: Delaney $T$, Wyse $R$, Yoong SL, et al. Cluster randomised controlled trial of a consumer behaviour intervention to improve healthy food purchases from online canteens: study protocol. BMJ Open 2017;7 e014569. doi:10.1136/ bmjopen-2016-014569

- Prepublication history for this paper is available online. To view these files please visit the journal online (http://dx.doi.org/10.1136/ bmjopen-2016-014569).

Received 4 October 2016 Revised 21 January 2017 Accepted 15 February 2017

\section{CrossMark}

For numbered affiliations see end of article.

\footnotetext{
Correspondence to

Tessa Delaney;

tessa.delaney@hnehealth.

nsw.gov.au
}

\section{ABSTRACT}

Introduction: School canteens represent an opportune setting in which to deliver public health nutrition strategies given their wide reach, and frequent use by children. Online school canteen ordering systems, where students order and pay for their lunch online, provide an avenue to improve healthy canteen purchases through the application of consumer behaviour strategies that impact on purchasing decisions. The aim of this study is to assess the efficacy of a consumer behaviour intervention implemented in an online school canteen ordering system in reducing the kilojoule, saturated fat, sugar and sodium content of primary student lunch orders.

Methods and analysis: The study will employ a cluster randomised controlled trial design. Approximately 1040 students (aged 5-12 years) from 10 primary schools in New South Wales, Australia, currently using an online canteen ordering system will be invited to participate. Schools will be randomised in a $1: 1$ ratio to receive either the intervention (enhanced system) or control (standard online ordering only). The intervention will include evidencebased strategies shown to influence healthy food purchasing (strategies targeting availability, menu labelling, placement and prompting). The primary outcomes of the trial will be the mean content per student online lunch order of (1) energy (kJ), (2) saturated fat $(\mathrm{g})$, (3) sugar $(\mathrm{g})$ and (4) sodium ( $\mathrm{mg}$ ). The impact of the intervention will be determined by between-group assessment of the nutritional content of lunch purchases over a 2-month period postintervention initiation.

Ethics and dissemination: The study was approved by the Hunter New England Human Research Ethics Committee, University of Newcastle Human Research Ethics Committee and New South Wales Department of Education and School Communities. Study findings will be disseminated widely through peer-reviewed publications and relevant presentations in international conferences and to stakeholders.

Trial registration number: ACTRN12616000499482.

\section{Strengths and limitations of this study}

The trial uses the most internally valid research design (randomised controlled trial), includes objective measures of trial outcomes, central randomisation to groups and has been powered to detect small but meaningful population-level intervention effects.

- The analysis of trial outcomes will be conducted by a statistician blinded to group allocation.

- The external validity of the findings may be limited, given the convenience sampling procedure and as the trial will be conducted in 10 schools from 1 Australian state.

\section{BACKGROUND}

Globally, poor diet (including excess intake of foods high in energy, saturated fat, added sugars and salt) is one of the leading causes of non-communicable diseases, including cardiovascular disease, type 2 diabetes and cancer. $^{1}{ }^{2}$ Given that dietary behaviours in childhood track into adulthood and are predictive of future non-communicable disease risk, ${ }^{3}$ improving child nutrition is a public health priority in Australia and internationally. ${ }^{1} 4$

Schools are a promising setting to improve child nutrition ${ }^{15}$ as they provide prolonged, centralised access to almost every child in high-income countries. Australian children consume almost $40 \%$ of their recommended energy intake while at school, ${ }^{4}$ with up to $72 \%$ of primary school-aged children purchasing their lunch from school canteens at least weekly. ${ }^{6}$ The foods ordered from such canteens however are typically high in energy, fat, sugar and salt, from products such as pies and sausage rolls (consumed regularly by $54 \%$ of students), pizza products $(30 \%)$, processed chicken $(29 \%)$ and hot 
$\operatorname{dogs}(18 \%) .^{7}$ Therefore, interventions to improve the nutritional quality of foods purchased at school canteens are a key opportunity to improve child nutrition. ${ }^{5}$

Findings of a recent systematic review of 18 school food environment studies suggest that modifying the relative availability of food for sale from school food services is associated with improvements in the purchase or consumption of healthy foods by students. ${ }^{8}$ Similarly, school-based research in the USA has found that a number of point of purchase strategies to influence consumer behaviour such as nutrition labelling, ${ }^{9}$ prompting $^{10}$ and the placement of menu items (including the order, prominence and access of items $)^{11}{ }^{12}$ are associated with the selection, purchase or consumption of healthier foods by students.

Online school ordering systems, which enable student school lunches to be ordered and paid for via the web, are becoming increasingly common in countries, including Australia (Personal communication with CEO Flexischools, March 2016) ${ }^{13}$ and the USA. ${ }^{14}{ }^{15}$ Such systems provide a platform to implement evidence-based consumer behaviour change strategies to support healthy purchasing choices by students and parents. Specifically, the online environment of these systems provides a controlled but dynamic infrastructure that enables implementation of a range of strategies that can reach large numbers of individuals at a relatively low cost. ${ }^{16}$ For example, point of purchase nutrition labelling, product placement and prompts, strategies that are routinely used by online food retailers to influence purchase decisions of consumers ${ }^{17}$ can be readily deployed to influence the purchase choices of a large number of students and parents. Despite the potential benefits of implementing these strategies to promote healthy online school canteen purchases, there have been no previous trials of their application to online school canteen ordering systems.

\section{Study aim}

In this context, the purpose of this study is to assess the efficacy of a consumer behaviour intervention implemented in an online school canteen ordering system in reducing the kilojoule, saturated fat, sugar and sodium content of primary student lunch orders.

\section{METHODS}

\section{Trial design}

The cohort study will employ a parallel group, cluster randomised trial design. Ten primary schools located in New South Wales, Australia, with an existing online canteen ordering system will be randomised to receive either a 2-month consumer behaviour intervention (enhanced system) or control (standard online ordering only). The efficacy of the intervention will be determined by assessing between-group differences at follow-up in the average (1) energy (kJ), (2) saturated fat (g), (3) sugar (g) and (4) sodium (mg) content of a cohort of students who had made an online lunch order during the baseline period. Student purchase data will be automatically collected by the online canteen system. Outcome data will be assessed at baseline and for the 2-month period following introduction of the intervention.

\section{Setting}

The study will take place in the state of New South Wales, Australia, a geographically large state including large metropolitan and non-metropolitan areas with a demographically and socioeconomically diverse population of $\sim 455000$ primary aged children and 1600 government primary schools. ${ }^{18}$ Children attend primary school from the age of 5 to the age of 12, with government schools being the major provider of school education $(65.2 \%) .{ }^{19}$

\section{Participants}

\section{Schools}

To be eligible, schools must be a government primary school in New South Wales with an operational canteen that has been using the online canteen ordering system supplied by a single specific provider (services $\sim 11 \%$ of New South Wales government school canteens (unpublished data) and henceforth referred to as 'the provider') for at least 6 months. In addition, schools must process a minimum of 50 student online lunch orders per month. Special purpose schools that exclusively enrol students with special needs, juvenile justice schools, schools serving hospitalised children or schools with externally licenced canteens will be excluded due to the potential differences in the provision of foods in these settings. A research assistant will screen the school's online menu, and any school already employing point of purchase nutrition labelling strategies (same as that of intervention) will be excluded.

\section{Students}

All users of the online school canteen ordering system (eg, children or parents ordering on behalf of their children) who place an online lunch order during the 2-month baseline data collection period will be eligible for study inclusion. Other users of the school's online canteen ordering system such as teaching staff, as identified by the online provider, will be excluded.

\section{Recruitment procedures}

A list of schools will be supplied by the provider and screened for eligibility by the research team. A convenience sample of $\sim 50$ schools currently using the online providers system will be invited to participate via mail and telephone with recruitment continuing until the required sample of schools $(\mathrm{N}=10)$ consent to participate. Schools that had been invited but were not within the first 10 schools to consent will not participate in the trial. The recruitment strategy will employ effective recruitment practices within the school setting. ${ }^{20}$ 
Specifically, one member of the research team will act as a dedicated recruitment coordinator. The coordinator will manage the recruitment of schools into the trial and monitor consent rates. Schools will be provided with the direct phone number of the trial manager for any enquiries regarding the research. Study information statements will be mailed to school Principals inviting study participation. Specifically, consent will be sought from the Principal for permission for the research team to access deidentified data regarding canteen lunch order purchases, user demographics and usage characteristics of the online ordering system. As deidentified student purchase data are accessed via a schoolcontrolled database, all data will be used and individual student consent will not be sought. Two weeks after sending the information statements, a research assistant will make multiple attempts to contact schools via the phone to confirm eligibility, answer any questions regarding the trial and invite participation. Following consent, the online provider will supply baseline lunch order purchase data (the 2-month operational period immediately preceding intervention initiation) of students, in a non-identifiable format, to the research team to assess the primary trial outcomes.

\section{Randomisation and blinding}

Following school recruitment, an independent statistician will use a computerised random number function in Microsoft Excel to randomise schools to either an intervention or a control group. Randomisation will occur at the unit of the school in a 1:1 (intervention: control) ratio in randomly sequenced blocks of two and four to ensure the number of schools allocated to each group remains approximately equal (see figure 1). ${ }^{21-23}$ Given evidence that socioeconomic status of the locality may be associated with the relative healthiness of student purchasing patterns, ${ }^{7}$ randomisation will be stratified by the socioeconomic status of a school locality based on school postcode. ${ }^{24}$ Owing to the difficulty in blinding the users of the online system to the changes introduced, the study will be conducted as an open trial; however, parent and student users will not specifically be informed of the experimental manipulation of the study. Furthermore, the study statistician undertaking the primary analyses will be blinded to group allocation.

\section{Intervention}

A consumer behaviour intervention will be integrated into the existing schools online canteen ordering system operated by the provider. Online canteen ordering systems allow users (students, or a parent on behalf of a student) to login to a website to access their school's lunch menu. Users are then able to select, order and pay for lunch items which are then processed by the canteen and supplied to students during their meal break. Research in food service settings suggests that decisions regarding food ordered for school-aged children are typically made jointly by parents and children. ${ }^{25}$ As such, the intervention seeks to encourage consumer (parent or child) purchase of healthier foods and beverages for school lunch orders, that is, food items lower in energy, saturated fat, sugar and/or sodium. All users of the online canteen ordering system at intervention schools will be exposed to the intervention. Contamination of intervention components between groups will be minimised by randomisation at the school level, and by the provider preventing user access to the intervention by control group schools.

\section{Intervention development and theoretical framework}

The intervention draws on the principles of choice architecture. $^{26}$ This approach posits that consumer behaviour is influenced by social and physical environments through conscious (eg, reflective) and unconscious (automatic) processes. ${ }^{26}$ Choice architecture strategies alter the environments in which decisions are made, in this case, to cue healthier purchasing choices. Consumer behaviour interventions, based on choice architecture, typically require minimal conscious engagement by the consumer and can include: the provision of information (prompting, labelling, feedback); altering of the physical environment (eg, altering the placement of products including accessibility, position or proximity, lighting, décor, etc) or the properties of products or stimuli within an environment (packaging, presentation, functionality) to cue desirable behaviour. ${ }^{26} 27$ The intervention component selection for this study was guided by the choice architecture typology proposed by Hollands et $a .^{26}$ Intervention selection was developed in consultation with a multidisciplinary team of experts, including health behaviour scientists, dietitians, canteen staff, parents, software providers and developers. The consumer behaviour strategies were included in the intervention if they were (1) supported by empirical evidence of beneficial effect in food-service settings; ${ }^{10} 2829$ (2) considered appropriate and acceptable to school principals $^{13}$ and parents ${ }^{30}$ and (3) able to be feasibly operationalised within the providers online ordering system.

\section{Intervention strategies}

The online provider will modify the display of the online ordering system to include the following evidence-based strategies that have previously been associated with healthier consumer choices in analogous settings (table 1). ${ }^{1028} 29$

\section{Availability}

Canteen managers will be supported by the research team to improve the relative availability of healthier items listed on their online canteen menu. Research in the school food setting has found a positive association between increased availability of healthy foods and improved purchasing behaviour of students. ${ }^{31}$ Therefore, a trained dietitian, experienced in canteen menu 


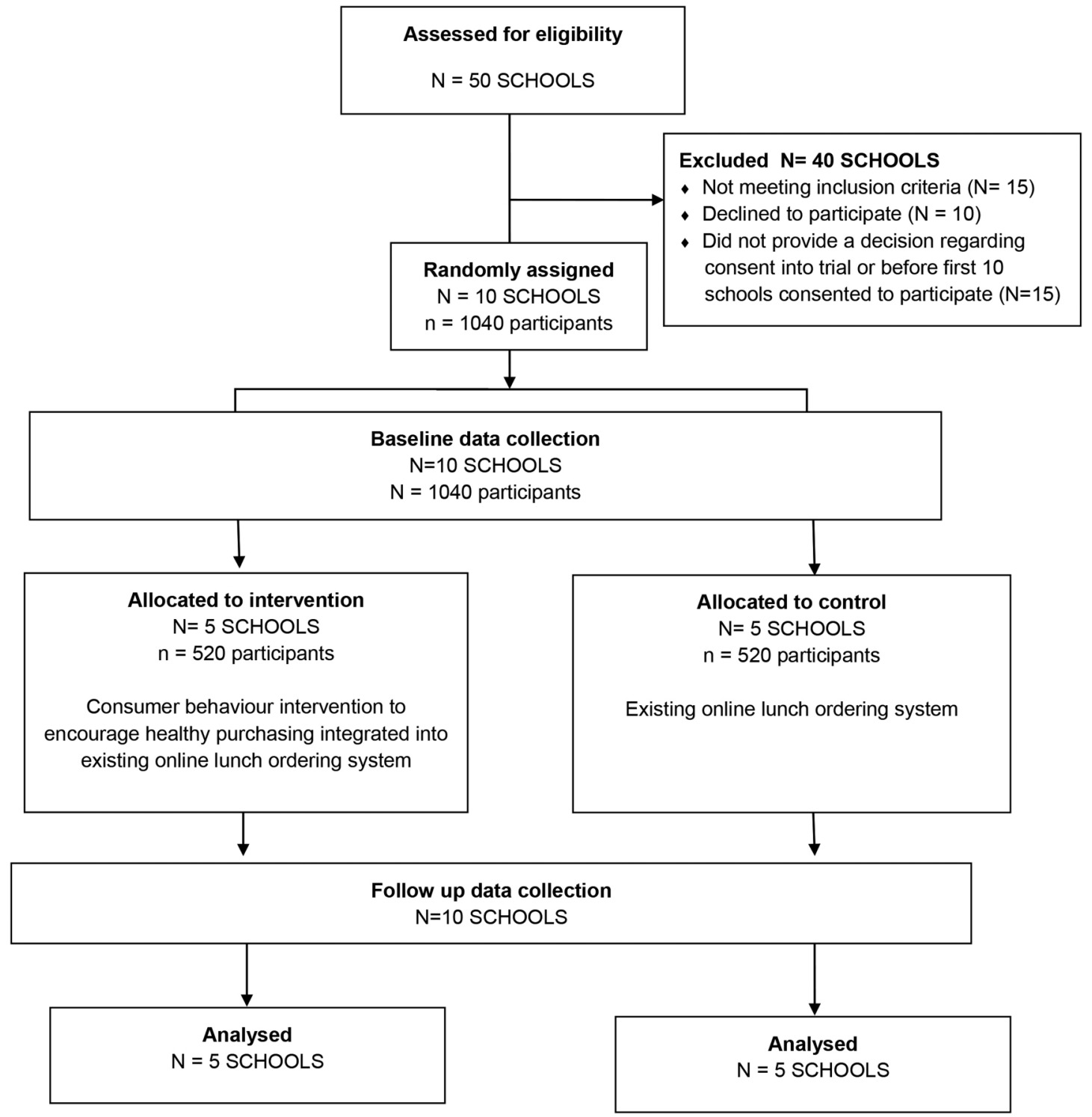

Figure 1 Estimated participant flow through trial. Numbers based on best available information at the time of submission.

assessment, will classify all foods and beverages listed on the canteen menu as 'red' (low in nutritional value), 'amber' (moderate nutritional value) or 'green' (high nutritional value) according to the New South Wales government school canteen policy 'Fresh Tastes @ School'. ${ }^{32}$ The dietitian will prepare and provide a comprehensive menu feedback report to schools encouraging canteen managers to improve the relative availability of healthy items by increasing the proportion of 'green' menu items (to $>50 \%$ of total items) or removing 'red' menu items. ${ }^{33}{ }^{34}$ Such strategies have been found to be effective in improving the relative availability of healthy items by school canteens. ${ }^{35}{ }^{36}$ These reports will be distributed once via email to the canteen manager and principal, immediately prior to the redesigned canteen menu being uploaded online. A brief phone call (of $\sim 15 \mathrm{~min}$ ) will be made to the canteen manager and/or principal to discuss contents of the feedback report.
Labelling

Labelling involves the application of written or graphical feedback or information endorsing a product at the point of purchase or point of choice. ${ }^{26}$ This strategy will comprise the following components within the online system:

\section{Traffic light labels}

A single red, amber or green circle will be added beside each menu item. ${ }^{37}$ The traffic light label will be based on Fresh Tastes @ School. ${ }^{32}$ The application of traffic light labels in hospital cafeterias has been shown to significantly decrease sale of less healthy and increase sale of healthier menu items. ${ }^{37}$ Traffic light labels, compared with other forms of labelling (eg, nutrient labelling), are more likely to be noticed by parents when making purchase decisions for their children from food settings. ${ }^{38}$ Furthermore, compared with other labelling systems, traffic light labels are preferred by adults and 
Table 1 Intervention strategies informed by Choice Architecture Framework proposed by Hollands et af6

\begin{tabular}{ll}
\hline Strategy & Description \\
\hline Availability* & Schools will receive a comprehensive feedback report \\
including strategies to improve the relative availability \\
of healthy foods including: \\
A colour-coded copy of their menu, \\
Graphical feedback comparing their menu to the \\
'Fresh Tastes' target of $>50 \%$ green and $0 \%$ red \\
- Tailored advice for how to amend the menu to be \\
compliant with the policy \\
Labelling Web links for additional support \\
Each menu item will display a single round traffic ligh
\end{tabular}
label

The menu will include information on how to use the label when selecting menu items (eg, 'best choice', 'select carefully', 'select occasionally')

Healthy menu items will include a hedonic description directly under the item name

Placement Healthy menu categories (eg, fruit, sandwiches, salads) and 'green' items within a category will be listed first

Healthy items (green) will be listed in main website interface

Placement: amber and red menu items with multiple flavours will require users to 'click'/explode the item before the full list of flavours are displayed

Prompting Amber and red hot item will include a prompt to add a healthy drink (water) or snack (fruit and/or veg) to the lunch order

Healthy food categories (eg, sandwiches, salad, fruit) will appear in bold font, have an image and a positive food prompt, eg, 'this is a good choice'

\section{Application}

If availability of items are modified by the school or canteen, this changes the capacity for user (parent or child) to select healthy vs less healthy foods

Provides nutritional information about the food at point of purchase

Promotes awareness of and thus motivation for a making a healthy purchase

Facilitates interpretation of label

Provides easily understood information about the healthiest choice

Provides incidental cues to induce non-conscious behavioural response (makes product more appealing) Makes healthy menu items more salient

Makes healthy menu items more salient, convenient and maximises opportunity for engagement with these items

Facilitates engagement with behavioural options by making healthy food more convenient/accessible Makes less healthy menu items harder to engage with therefore less convenient/accessible

Raises awareness of a given behaviour

Makes it easier to access healthy food options

Provides general encouragement

Promotes motivation for a given behaviour

*This strategy aims to increasing healthy foods and involves providing the school with feedback on how to restrict availability of unhealthy foods in line with Fresh Tastes @ School policy.

children, ${ }^{39}$ are more easily understood and more effective in helping consumers to correctly identify healthier food products. ${ }^{40}$

\section{Label guide}

An explanation of the relative healthiness of 'green', 'amber' and 'red' foods ${ }^{28}{ }^{37}$ will appear at the top of the online canteen website and will pop up when a user hovers their cursor over each traffic light label.

\section{Descriptions}

'Green' menu items that require onsite preparation (eg, salads, sandwiches, homemade hot meals) will receive an appealing description directly under item name (eg, 'super salad tub'). Research in the restaurant setting has demonstrated that creative descriptions applied to menu items have been associated with an increase in sales by up to $27 \% .{ }^{41}$ Research in the school setting has similarly shown that adding creative names to healthy food items (eg, 'X-ray vision carrots') is associated with increases in children's consumption of the item. ${ }^{9}$

\section{Placement}

Placement strategies will be employed to alter (1) the position of menu items to make them appear more immediately prominent and (2) the accessibility of menu items to make healthier choices easier to select and less healthy choices harder to select. ${ }^{26}$ Evidence suggests that items that are placed at the beginning or the end of the menu section were selected up to twice as frequently as when they were placed in the centre of the list. ${ }^{29}$ Therefore, healthier menu categories (ie, fruit, sandwiches, salads) and items within categories will be ordered to give healthy items positions of greatest prominence; that is, 'green' items will be positioned first; 'red' items will be located in the middle and 'amber' items will be positioned last in a food list. Where there are multiple flavours of a 'red' or 'amber' food, users will be required to first 'click' on the category before the 
full list of items are displayed. For example, for a user to select a flavour of potato crisps, they will first be required to click on that product category ('crisps'), then select their preferred flavour in a separate pop up box. Conversely, all available flavours of 'green' items will appear in the main website interface without requiring further selection actions.

\section{Prompting}

Standardised written and graphical information intended to promote or raise the awareness of, or the motivation for a given behaviour will be included in the online menu. Motivational written and graphical prompts will be used to promote and encourage selection of healthy items. For example, healthier menu categories (ie, sandwiches, salads, fruit) will be accompanied by positive purchase prompts (eg, 'This is a good choice') and an appealing image representing the category. ${ }^{10}$ When users select a red or amber hot food item, they will also be prompted with a list of green menu items, 'meal extras', which typically include bottled water, fresh fruit or vegetable pieces, to add to their order.

Once implemented, the intervention will remain operational across the entire study period.

\section{Intervention integrity}

A dietitian will use the colour-coded menu items to redesign the menu for online display using a standardised template ('menu template'). This template has been pilot tested and refined based on feedback by the dietitian and the provider of the online lunch ordering system. The completed menu template will be sent via email to the provider who will 'upload' the schools online menu as per specifications in the menu template. This process will be managed centrally by the provider. After the menu is uploaded but prior to being operationalised, the research team will be able to view the redesigned menu in order to confirm that the strategies have been applied and uploaded correctly. In order to monitor and manage intervention integrity, once the redesigned menu is operational, the provider will supply the research team with two reports (start and midintervention) listing any changes that have been made to the online menu by the school. These reports will enable the research team to identify new menu items that have been added. The research team will then label and position new menu items according to the menu template and contact the provider to make the required changes.

\section{Control group}

Schools allocated to the control group will continue to receive the standard online lunch ordering service and will not have access to the intervention until after follow-up data collection at which point they will be offered access to supportive strategies.

\section{DATA COLLECTION PROCEDURES AND MEASURES}

\section{Primary outcomes}

The primary trial outcomes are: the mean content per student online lunch order of (1) energy (kJ), (2) saturated fat $(\mathrm{g})$, (3) sugar $(\mathrm{g})$ and (4) sodium (mg). Given the effect of similar interventions has been reported to be immediate, ${ }^{42}$ the primary trial end point is 2 months postintervention initiation (during which the canteen is operational). Primary trial outcomes will be collected at baseline (the 2-month operational period immediately preceding intervention initiation) and follow-up (the 2-month period operational period postintervention initiation). Data from all purchases occurring during the baseline and follow-up assessment periods for the cohort of students will be used to determine the trial outcomes. No assessment of plate waste will be conducted. Purchase data have been shown to be highly correlated with food consumed. ${ }^{43}$

Data collection procedures will be in accordance with previous canteen trials conducted by the research team. ${ }^{33}$ Specifically, a dietitian will contact the canteen manager over the phone to obtain nutrition information of canteen menu items available online. For prepackaged menu items, the canteen manager will be asked to specify brand name, product name and serve size. The nutritional profile of each prepackaged item will be obtained by searching the 'brand', 'product name' and 'serve size' in a canteen product database consisting of over 1300 commonly stocked school canteen items developed by the research team. ${ }^{44}$ If the menu item is not listed in the canteen product database, the dietitian will use a publicly available database of commercial items (Foodswitch) to obtain the nutrition information panel. ${ }^{45}$ If the item cannot be located in either database, the dietitian will contact the manufacturer to obtain the nutrition information panel. If the dietitian cannot obtain the nutrition information panel from the manufacturer, a 'generic' nutrient profile will be assigned using a commercial equivalent found in the canteen product database.

For menu items that are not packaged (eg, freshly made foods such as sandwiches, canteen made hot foods and snacks), dietitians will request a copy of the recipe from the canteen manager, including recipe yield, ingredients and serve size. Dietitians will then use a commercially available Australian nutrition database (Foodworks) (Foodworks, version 7, Xyris Software, Highgate Hill, Australia. https://www.xyris.com.au/) to create a nutrient profile for this item (eg, a ham, cheese and tomato sandwich). In the absence of a complete recipe, a 'generic' nutrient profile will be created using a commercial equivalent found in the canteen product database. Detailed records will be maintained for all items (prepackaged and freshly prepared) that required a 'generic' nutrient profile to be assigned.

Using the nutritional profile data, a dietitian will determine the nutrient profile (kilojoules, saturated fat, sugar, sodium) and Fresh Tastes classification (red, amber, green $)^{32}$ for each menu item. 
To enable calculation of the primary trial outcomes, the nutrition profile for each menu item will be applied to purchasing data obtained by the provider to generate a nutritional profile for each individual order placed. A unique deidentified numerical identifier by the provider will be used to link student orders across and within baseline and follow-up data collection periods.

\section{Secondary outcomes}

Nutrition quality

(1) The proportion of all student lunch orders that are (i) green and (ii) red; and (2) the mean per cent of energy of lunch orders from (i) sugar; and (ii) saturated fat per student online lunch order will be collected at baseline (the 2-month operational period immediately preceding intervention initiation) and follow-up (the 2-month operational period postintervention initiation) and compared between groups at follow-up. The colour code and per cent energy from saturated fat and sugar will be based on the dietitian's nutritional assessment of the purchasing data recorded by the online ordering system (described above). Conversion of sugar and saturated fat to energy will be based on internationally accepted conversion factors of 17 and $37 \mathrm{~kJ} / \mathrm{g}$, respectively. ${ }^{46}$

\section{Revenue}

Revenue data will be automatically collected and supplied by the online provider. The average weekly online canteen revenue will be assessed at baseline (the 2-month operational period immediately preceding intervention initiation) and follow-up (the 2-month operational period postintervention initiation). The average weekly online canteen revenue will be compared between groups to assess any detrimental or beneficial impact of the intervention on school revenue that may affect the sustainability of the intervention.

\section{Other data}

\section{School characteristics}

School-level data, including school size (number of enrolments), year range (eg, Kindergarten to grade 6) and school postcode, will be collected from the "My School' website. ${ }^{47}$

\section{User characteristics}

Child school grade and the recorded user (parent or child) will be collected from the online ordering system. Online canteen usage data (eg, frequency of placing an order, the device used to place the order, the time taken to place the order) are automatically collected by the system, and will also be accessed by the research team.

\section{Canteen manager survey}

After the collection of follow-up purchasing data (2 months operational period postintervention), canteen managers will be contacted to take part in a telephone survey to determine (1) canteen characteristics (type of canteen operation (leased, P\&C run, school run); staffing (paid or unpaid), profit) and (2) the acceptability of the intervention strategies using a four-point Likert scale from 'strongly agree' to 'strongly disagree'.

School characteristics, user characteristics and canteen manager survey data will be collected and used for descriptive purposes.

\section{Availability of menu items}

(1) The proportion of 'green' items available on the menu and (2) the proportion of 'red' items available on the menu will be assessed at baseline (immediately prior to intervention initiation) and follow-up (2-months postintervention initiation). Copies of each school's canteen menu will be obtained during baseline data collection period (immediately after the school consents into the trial) and on the last day of the follow-up data collection period. Each menu will be independently audited by two dietitians consistent with previous studies. ${ }^{33}$ The menu audit procedure will involve assigning each item a colour-code (as per the Fresh Tastes @ School guidelines) and calculating the proportion of each colour on the menu, in accordance with procedures previously described elsewhere. ${ }^{33} 34$ Any discrepancies between dietitians in assigning a colour code or calculating the proportion of green or red items available on the menu will be resolved through consensus processes.

\section{ANALYSIS AND SAMPLE SIZE Analysis}

The analyses will be undertaken by a statistician blinded to group allocation, with no other involvement in the trial. Intervention effectiveness will be assessed using a separate linear mixed model ${ }^{48}$ for each primary outcome under an intention-to-treat approach: ${ }^{49}$ energy $(\mathrm{kJ})$, saturated fat $(\mathrm{g})$, sodium $(\mathrm{mg})$ and sugar $(\mathrm{g})$. The analysis of primary outcomes will be conducted only after completion of final follow-up data collection and no interim analyses of trial outcomes will be performed. The average nutritional content (eg, mean kilojoule content) will be calculated across all online lunch orders placed by a student during the follow-up data collection period and compared between the intervention and control groups, adjusting for clustering at the school level and controlling for baseline values. The mixed model will account for repeated measures of the trial outcome at the student and school level. Adjusting for baseline will control for known and unknown potential confounders as any differences in prognostic factors at baseline will be captured in the baseline values for energy, fat, sugar and sodium. All students that place an order during the baseline period will be included in the primary analysis. Missing data could arise at follow-up due to a student not placing an online lunch order during the follow-up period. Multiple imputation will be used for any missing data at follow-up as recommended by White et $a \tilde{l}^{0}$ as part of a sensitivity analysis. 
Exploratory subgroup analyses will also be conducted, testing for treatment group interactions by demographic (ie, student grade) and purchasing characteristics of the sample.

The trial data will be reported in adherence with the CONSORT 2010 guidelines for reporting clustered randomised controlled trials. The trial has been prospectively registered with the Australian New Zealand Clinical Trials Registry ACTRN12616000499482.

\section{Sample size calculation}

Given there are dose-response relationships between intake of saturated fat, ${ }^{51}$ sugar $^{52}$ and sodium ${ }^{53}$ and important clinical health outcomes, including precursors for chronic disease (such as blood pressure), the sample size calculation was conducted based on estimated changes in energy intake between groups where a reduction in a defined magnitude is required to accrue health benefit at the population level. Specifically, a reduction of 192-300 kJ of energy per day is estimated to offset overweight in children ${ }^{54}$ and in doing so reduce population level risk for chronic disease. Assuming that 104 students per school place at least 1 online lunch order over the data collection period, and assuming that a standard student lunch order contains $1729 \mathrm{~kJ}(\sim 25 \%$ total daily energy intake $\left.{ }^{4}\right) \quad(\mathrm{SD}=700) \quad$ (unpublished data from research team) with an ICC of 0.05 , the participation of 10 schools ( 5 each arm) in the trial would enable detection of $\sim 300 \mathrm{~kJ}$, difference between groups at follow-up with $80 \%$ power at the 0.05 significance level. A change of this magnitude is considered clinically meaningful to detect a change in population body weight. ${ }^{54-56}$

\section{DISCUSSION}

This will be the first study to examine the efficacy of a consumer behaviour intervention implemented in an online school canteen ordering system on purchasing behaviour from primary school canteens and will represent a substantial advance in knowledge in the field of school-based public health nutrition. Further, given that online interventions can be delivered to large numbers of community members at relatively low cost, the intervention, if effective, may represent an attractive strategy to contribute to improvements in child health and reductions in chronic disease risk.

While the trial will provide useful information for policymakers and practitioners, and valuable data for future studies examining technology-based nutrition interventions in the school setting, there are a number of study limitations. First, the trial uses convenience sampling methods, and is conducted using one provider of online school canteen ordering systems in Australia, limiting the external validity of trial findings. Furthermore, the trial tests a complex public health intervention and is not designed to assess the independent effects of individual strategies used in the intervention. Future research using factorial designs would be warranted if the intervention is found to improve child diet in order to understand intervention mechanisms and to design more efficient interventions in the future.

\section{DISSEMINATION}

Modifications to the trial protocol will be made via the Australian and New Zealand Clinical Trials Registry and outlined in the final publication. Evaluation data and process data collected as part of the study may be presented at scientific conferences, be published within scientific journals and form part of student theses. Participant's confidentiality will be maintained.

\section{Author affiliations}

${ }^{1}$ Hunter New England Population Health, Wallsend, New South Wales, Australia

${ }^{2}$ School of Medicine and Public Health, University of Newcastle, Callaghan, New South Wales, Australia

${ }^{3}$ Hunter Medical Research Institute, Newcastle, New South Wales, Australia ${ }^{4}$ Priority Research Centre for Health Behaviour, The University of Newcastle, New South Wales, Australia

${ }^{5}$ Institute for Physical Activity and Nutrition (IPAN), School of Exercise and Nutrition Sciences, Deakin University, Burwood, Victoria, Australia

${ }^{6}$ NSW Office of Preventive Health, and The University of Sydney, Sydney, New South Wales, Australia

Acknowledgements The authors wish to acknowledge Christophe Lecathelinais for statistical advice and Flexischools for enabling the research to be undertaken using their online canteen ordering infrastructure.

Contributors TD led the development of this manuscript. RW, TD and LW conceived the intervention concept. JW, LW, RW, RS, SLY, KC, KB, CR and TD contributed to research design and trial methodology. All authors contributed to and approved the final version of this manuscript.

Funding Inkind funding was received by Hunter New England Population Health and University of Newcastle Australia to support conduct of the trial. Infrastructure support was also provided by Hunter Medical Research Institute. Inkind support for the delivery of the intervention was provided by Flexischools (provider of online canteens). RW is a research fellow funded through the National Health and Medical Research Council's Translating Research into Practice Scheme. SLY is a postdoctoral research fellow funded by the National Heart Foundation. KB is a National Health and Medical Research Council (NHMRC) Principal Research Fellowship (1042442). LW is a National Health and Medical Research Council Career Development Fellow.

Disclaimer The contents of this manuscript are the responsibility of the authors and do not reflect the views of the NHMRC.

Competing interests The online provider (Flexischools) was selected through a competitive tender process. Flexischools is a commercial organisation which provided online canteen ordering infrastructure to schools included in the study.

Ethics approval Hunter New England Human Research Ethics Committee (reference: 06/07/26/4.04), University of Newcastle (Ref. No. H-2008-0343), NSW Department of Education and Communities (SERAP 2012277).

Provenance and peer review Not commissioned; externally peer reviewed.

Data sharing statement Ultimate authority over the publication rests with the primary author. Access to the trial data set and full protocol will be available after publication of the study findings and on request to the primary author.

Open Access This is an Open Access article distributed in accordance with the Creative Commons Attribution Non Commercial (CC BY-NC 4.0) license, which permits others to distribute, remix, adapt, build upon this work noncommercially, and license their derivative works on different terms, provided the original work is properly cited and the use is non-commercial. See: http:// creativecommons.org/licenses/by-nc/4.0/ 


\section{REFERENCES}

1. World Health Organisation. Global Strategy on Diet, Physical Activity and Health. 2004. http://www.who.int/dietphysicalactivity/goals/en/ index.html. (accessed 8 Dec 2013).

2. World Health Organization. Diet, nutrition and the prevention of chronic diseases. World Health Organ Tech Rep Ser 2003;916:i-viii. 1-149, backcover.

3. Maynard M, Gunnell D, Emmett P, et al. Fruit, vegetables, and antioxidants in childhood and risk of adult cancer: the Boyd Orr cohort. J Epidemiol Community Health 2003;57:218-25.

4. Bell AC, Swinburn BA. What are the key food groups to target for preventing obesity and improving nutrition in schools? Eur J Clin Nutr 2004;58:258-63.

5. World Health Organization. Population-based approaches to childhood obesity prevention. Geneva: World Health Organisation, 2012.

6. Hardy L, King L, Espinel P, et al. NSW Schools Physical Activity and Nutrition Survey (SPANS) 2010: Full Report. Sydney: NSW Ministry of Health, 2011.

7. Finch $M$, Sutherland $R$, Harrison $M$, et al. Canteen purchasing practices of year 1-6 primary school children and association with SES and weight status. Aust N Z J Public Health 2006;30:247-51.

8. Driessen CE, Cameron AJ, Thornton LE, et al. Effect of changes to the school food environment on eating behaviours and/or body weight in children: a systematic review. Obes Rev 2014;15:968-82.

9. Wansink B, Just DR, Payne CR, et al. Attractive names sustain increased vegetable intake in schools. Prev Med 2012;55:330-2.

10. Schwartz MB. The influence of a verbal prompt on school lunch fruit consumption: a pilot study. Int J Behav Nutr Phys Act 2007;4:6.

11. Hanks AS, Just DR, Smith LE, et al. Healthy convenience: nudging students toward healthier choices in the lunchroom. J Public Health (Oxf) 2012;34:370-6.

12. Hanks AS, Just DR, Wansink B. Smarter lunchrooms can address new school lunchroom guidelines and childhood obesity. J Pediatr 2013;162:867-9.

13. Wyse R, Yoong SL, Dodds $\mathrm{P}$, et al. Online canteens: awareness, use, barriers to use, and the acceptability of potential online strategies to improve public health nutrition in primary schools. Health Promot J Austr 2016;28:67-71.

14. Order Lunches-About Us 2017. http://www.orderlunches.com/ about-us/ (accessed 16 Jan 2017).

15. Nutrilink Technologies 2017. http://www.nutri-linktechnologies.com/ company/ (accessed 16 Jan 2017).

16. Norman GJ, Zabinski MF, Adams MA, et al. A review of eHealth interventions for physical activity and dietary behavior change. Am J Prev Med 2007;33:336-45.e16.

17. Wylie N, Dougan M, Harpur P, Clarke A. The rapid growth of online shopping is driving structural changes in the retail market. Frost \& Sullivan and PricewaterhouseCoopers. 2012.

18. NSW Department of Education. Schools and students: 2014 statistical bulletin. Sydney: Centre for Education Statistics and Evaluation, 2014.

19. Australian Bureau of Statistics. 4221.0-Schools, Australia. Canberra: AUS: Australian Bureau of Statistics (ABS), 2015. http:// www.abs.gov.au/ausstats/abs@.nsf/mf/4221.0

20. Wolfenden L, Kypri K, Freund M, et al. Obtaining active parental consent for school-based research: a guide for researchers. Aust $N$ Z J Public Health 2009;33:270-5.

21. Efird J. Blocked randomization with randomly selected block sizes. Int J Environ Res Public Health 2011;8:15-20.

22. Suresh KP. An overview of randomization techniques: an unbiased assessment of outcome in clinical research. J Hum Reprod Sci 2011;4:8-11.

23. Altman DG, Bland JM. How to randomise. BMJ 1999:319:703-4.

24. Australian Bureau of Statistics. Technical paper: socio-economic indexes for areas. Canberra: Australian Bureau of Statistics, 2011.

25. Wellard L, Chapman K, Wolfenden L, et al. Who is responsible for selecting children's fast food meals, and what impact does this have on energy content of the selected meals? Nutr Diet 2014;71:172-7.

26. Hollands GJ, Shemilt I, Marteau TM, et al. Altering micro-environments to change population health behaviour: towards an evidence base for choice architecture interventions. BMC Public Health 2013;13:1218

27. Bucher T, Collins C, Rollo ME, et al. Nudging consumers towards healthier choices: a systematic review of positional influences on food choice. Br J Nutr 2016;115:2252-63.

28. Thorndike AN, Riis J, Sonnenberg LM, et al. Traffic-light labels and choice architecture: promoting healthy food choices. Am J Prev Med 2014;46:143-9.

29. Dayan E, Bar-Hillel M. Nudge to nobesity II: menu positions influence food orders. Judgm Decis Mak 2011;6:333.
30. Wyse R, Delaney T, Nathan N, et al. eLunch? Will parents use online canteens to purchase their child's lunch? Australian \& New Zealand Obesity Society 2015 Annual Scientific Meeting, 2015.

31. Jaime PC, Lock K. Do school based food and nutrition policies improve diet and reduce obesity? Prev Med 2009;48:45-53.

32. NSW Department of Health, NSW Department of Education and Training. 'Fresh Tastes @ School' NSW healthy school canteen strategy: canteen menu planning guide. 3rd edn. Sydney: NSW Department of Health and NSW Department of Education and Training, 2006.

33. Wolfenden L, Nathan N, Williams CM, et al. A randomised controlled trial of an intervention to increase the implementation of a healthy canteen policy in Australian primary schools: study protocol. Implement Sci 2014;9:147.

34. Williams CM, Nathan N, Delaney T, et al. CAFE: a multicomponent audit and feedback intervention to improve implementation of healthy food policy in primary school canteens: protocol of a randomised controlled trial. BMJ Open 2015;5:e006969.

35. Wolfenden L, Nathan N, Janssen LM, et al. Multi-strategic intervention to enhance implementation of healthy canteen policy: a randomised controlled trial. Implement Sci 2017;12:6.

36. Nathan N, Yoong SL, Sutherland R, et al. Effectiveness of a multicomponent intervention to enhance implementation of a healthy canteen policy in Australian primary schools: a randomised controlled trial. Int J Behav Nutr Phys Act 2016;13:106.

37. Thorndike AN, Sonnenberg L, Riis J, et al. A 2-phase labeling and choice architecture intervention to improve healthy food and beverage choices. Am J Public Health 2012;102:527-33.

38. Dodds $\mathrm{P}$, Wolfenden L, Chapman K, et al. The effect of energy and traffic light labelling on parent and child fast food selection: a randomised controlled trial. Appetite 2014;73:23-30.

39. Talati Z, Pettigrew S, Kelly B, et al. Consumers' responses to front-of-pack labels that vary by interpretive content. Appetite 2016;101:205-13

40. Kelly B, Hughes C, Chapman K, et al. Consumer testing of the acceptability and effectiveness of front-of-pack food labelling systems for the Australian grocery market. Health Promot Int 2009;24:120-9.

41. Wansink B, Painter J, Ittersum KV. Descriptive menu labels' effect on sales. Cornell Hotel Restaur Adm Q 2001;42:68-72.

42. Chu YH, Frongillo EA, Jones SJ, et al. Improving patrons' mea selections through the use of point-of-selection nutrition labels. Am J Public Health 2009;99:2001-5

43. Ransley JK, Donnelly JK, Khara TN, et al. The use of supermarket till receipts to determine the fat and energy intake in a UK population. Public Health Nutr 2001;4:1279-86.

44. Reilly K, Nathan N, Wolfenden L, et al. Validity of four measures in assessing school canteen menu compliance with state-based healthy canteen policy. Health Promot $J$ Austr 2016;27:215-21.

45. FoodSwitch by The George Institute. http://www.foodswitch.com.au/

46. Kraisid T, MacLean W, Warwick P. Food energy: methods of analysis and conversion factors: report of a technical workshop, Rome, 3-6 December 2002. Rome: FAO, 2003.

47. My School. 2016. https://www.myschool.edu.au/

48. Stroup WW. Generalized linear mixed models: modern concepts, methods and applications. CRC Press, 2012.

49. Gupta SK. Intention-to-treat concept: a review. Perspect Clin Res 2011:2:109-12.

50. White IR, Horton NJ, Carpenter J, et al. Strategy for intention to treat analysis in randomised trials with missing outcome data. BMJ 2011;342:d40.

51. Schwab U, Lauritzen L, Tholstrup T, et al. Effect of the amount and type of dietary fat on cardiometabolic risk factors and risk of developing type 2 diabetes, cardiovascular diseases, and cancer: a systematic review. Food Nutr Res 2014. doi:10.3402/fnr.v58.25145. [Epub ahead of print 10 Jul 2014].

52. Basu S, Yoffe P, Hills N, et al. The relationship of sugar to population-level diabetes prevalence: an econometric analysis of repeated cross-sectional data. PLoS ONE 2013;8:e57873.

53. He FJ, Li J, MacGregor GA. Effect of longer term modest salt reduction on blood pressure: Cochrane systematic review and meta-analysis of randomised trials. BMJ 2013;346:f1325.

54. Plachta-Danielzik S, Landsberg B, Bosy-Westphal A, et al. Energy gain and energy gap in normal-weight children: longitudinal data of the KOPS. Obesity (Silver Spring) 2008;16:777-83.

55. Hall KD, Sacks G, Chandramohan D, et al. Quantification of the effect of energy imbalance on bodyweight. Lancet 2011;378:826-37.

56. Wang YC, Orleans CT, Gortmaker SL. Reaching the healthy people goals for reducing childhood obesity: closing the energy gap. Am J Prev Med 2012:42:437-44 